\title{
Using a runway paradigm to assess the relative strength of rats' motivations for enrichment objects
}

\author{
Louise A. Hanmer, Patricia M. Riddell, and Claire M. Williams \\ University of Reading, Reading, England
}

\begin{abstract}
Laboratory animals should be provided with enrichment objects in their cages; however, it is first necessary to test whether the proposed enrichment objects provide benefits that increase the animals' welfare. The two main paradigms currently used to assess proposed enrichment objects are the choice test, which is limited to determining relative frequency of choice, and consumer demand studies, which can indicate the strength of a preference but are complex to design. Here, we propose a third methodology: a runway paradigm, which can be used to assess the strength of an animal's motivation for enrichment objects, is simpler to use than consumer demand studies, and is faster to complete than typical choice tests. Time spent with objects in a standard choice test was used to rank several enrichment objects in order to compare with the ranking found in our runway paradigm. The rats ran significantly more times, ran faster, and interacted longer with objects with which they had previously spent the most time. It was concluded that this simple methodology is suitable for measuring rats' motivation to reach enrichment objects. This can be used to assess the preference for different types of enrichment objects or to measure reward system processes.
\end{abstract}

Improving the welfare of captive animals, particularly that of laboratory animals, is an important issue. A common method used to increase welfare is to provide caged animals with enrichment items that allow them to perform important natural behaviors (e.g., chewing, foraging, nesting). Environmental enrichment (EE) implies that the items introduced into the cage will have a positive effect on the welfare of the animals-for instance, by increasing nesting, exploration, or play behavior. Currently, cages may be enriched, often with some bedding material (Bradshaw \& Poling, 1991; Heizmann, Jonas, Hirschenauer, \& Havelec, 1998; Patterson-Kane, 2003; Townsend, 1997; Van de Weerd, Van Loo, Van Zutphen, Koolhaas, \& Baumans, 1998a, 1998b) or tubes on which rats can chew (Bradshaw \& Poling, 1991; Chmiel \& Noonan, 1996; Sørensen, Krohn, Hansen, Ottesen, \& Hansen, 2005). Although in the U.K., it is a requirement that Home Office license holders provide some form of enrichment for caged animals, the degree of provision and the types of objects used are not uniform across laboratories. This is partly due to the lack of information available to determine which provisions are most useful. Hence, a simple methodology that can be used to determine preference for EE objects would be of use in the process of standardizing EE provision.

EE researchers often attempt to assess an animal's motivation for a particular stimulus either by measuring the choice(s) that the animal makes when faced with two or more different stimuli or by measuring the time that the animal spends with each stimulus (e.g., Chmiel \& Noonan, 1996; Heizmann et al., 1998; Van de Weerd et al., 1998b). However, motivational experiments can also be used to judge preference by requiring the animals to actively work for objects - for example, by requiring the animals to press a lever in order to gain access to the enrichment (e.g., Sherwin \& Nicol, 1997). This method assumes that animals will be more motivated to work for some objects, thus enabling a rank order for motivational preference to be established. The present study was designed to determine whether a simple runway paradigm could be used to assess the strength of rats' motivation to run to different enrichment objects, thus providing a measure of preference based on the rats' motivation to work (i.e., run down the runway) for a particular object. This paradigm would improve on previous passive measures, since these measures require only that rats interact with an object that is placed in their environment. In addition, it would improve on consumer demand designs in being simpler to design and quicker to run.

Typically, in a runway paradigm, a food reward (i.e., sucrose solution) is placed in the reward box at the end of the runway; a rat is placed in the start box and released, at which point it runs to gain access to the palatable drink. Consistent findings show that as the drink is made progressively sweeter, the rats run faster to reach it, indicating that it is more rewarding and that the rats are more strongly motivated to reach it. When the concentration of the sucrose solution is reduced and it is therefore made less

C. M.Williams, claire.williams@rdg.ac.uk 
rewarding, the rats are found to run more slowly or fewer times, indicating a lower motivation to reach it (Burns \& Griner, 1993; Burns, Ziropadja, \& Djuric, 1984; Flaherty, Riley, \& Spear, 1973).

The runway paradigm was adapted to assess rats' relative motivation to run for enrichment objects instead of food, through placement of an enrichment object at one end of the runway and the rat at the other end. The rats then had to work, by running the length of the runway in order to be able to interact with the object. It was assumed that the rats would be more highly motivated to reach an object that they had previously ranked high, as opposed to an object that had been ranked low. This would be indicated by the rats' running more often, running faster, and/ or interacting longer with the top-ranked object. Because of the nature of the runway paradigm, the rats were only allowed to interact with the enrichment object for a short time upon reaching it (5 sec in Experiment 1 and $10 \mathrm{sec}$ in Experiment 2). This was in order to maintain the rats' motivation to gain access to the object, since if the rats were given as much time as they liked to interact with the object on the first encounter, they might be less motivated to run on subsequent trials. Despite the short length of time permitted to interact with the object, the rats still had the chance to choose to interact longer with some objects than with others over the course of the experiment, providing a ranking for the objects.

In the first experiment, two objects that had previously been ranked at the top and bottom in an open-field paradigm were tested. We predicted that the rats would run more often and faster to the top-ranked object than to the bottom-ranked object and that they would interact longer with the top-ranked object when they reached it.

\section{EXPERIMENT 1}

In order to determine whether the runway paradigm was suitable for testing motivational preferences for enrichment objects, we used objects for which a rank order had previously been determined by measuring the time spent with the object in an open field (Williams, Riddell, \& Scott, 2008). In the first experiment, objects with which rats had previously spent a lot of time (top ranked) or very little time (bottom ranked) were selected for assessment in the runway. This resulted in two sets of objects: one pair consisting of a top-ranked plastic house and a bottomranked cardboard tube (the EE set) and one pair consisting of a top-ranked large block made from plastic Lego bricks and a bottom-ranked single Lego brick covered in synthetic fur (the Lego set).

Two replications were carried out with each set of objects in order to thoroughly check that this methodology provided consistent rankings and to determine whether they were the same as those that had previously been recorded using time spent with the object. These replications confirmed that the rats would run faster and more often to a top-ranked object, but ceiling effects prevented demonstration that the rats would also interact longer with the top-ranked object. In Experiment 2, we increased the length of time available to interact with a set of objects and demonstrated that this parameter also differentiated between top- and bottom-ranked objects, with the rats interacting longer with a top-ranked object.

\section{Method}

Subjects. Adult male Lister hooded rats $(N=18)$, weighing 460 $600 \mathrm{~g}$, were group housed in standard cages under standard conditions $(n=4$ rats per cage). Each cage measured $23(\mathrm{H}) \times 32(\mathrm{~W}) \times$ $52 \mathrm{~cm}(\mathrm{~L})$, giving a total available floor space of $1,664 \mathrm{~cm}^{2}$. The cages were solid bottomed and contained both sawdust and a large cardboard tube. The cages were kept in a temperature- $\left(21^{\circ} \mathrm{C} \pm 1{ }^{\circ} \mathrm{C}\right)$ and humidity- $(55 \% \pm 10 \%)$ controlled environment under a reversed 12:12-h light:dark cycle (lights off at 10:00). The animals had ad lib access to food (PCD Mod C; Special Diet Services, Witham, U.K.) and water, except during testing. All testing was conducted during the dark phase under red light.

Apparatus. A runway, constructed from wood, measuring $185 \mathrm{~cm}$ long, with a $26 \times 24 \mathrm{~cm}$ reward area at one end, was used (Figure 1). The first $39 \mathrm{~cm}$ formed the start box, separated from the main runway by a plastic door. The walls of the entire apparatus were $19 \mathrm{~cm}$ high and were covered with a clear Perspex lid to prevent the rats from climbing out.

Objects. One object was used to train the rats and to collect baseline data. This was a plastic ball-shaped object $(36-\mathrm{cm}$ circumference), with holes around the sides (5 $\mathrm{cm}$ in diameter; see Figure 2A). The two sets of objects used in testing consisted of one top-ranked and one bottom-ranked object, as was determined by previously published experimental data (Williams et al., 2008). Two sets of objects were prepared for this study.

The Lego set consisted of objects made from Lego bricks (Figure $2 \mathrm{~B}$ ). This set consisted of a large plastic block made from Lego bricks $(9.5 \times 6 \times 5 \mathrm{~cm})$, which had been the top-ranked object in the

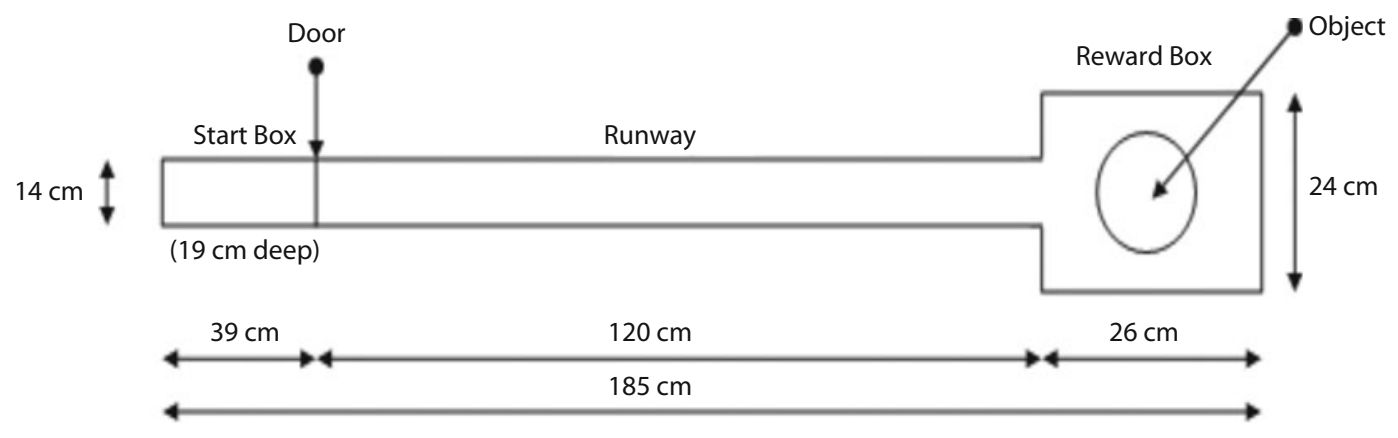

Figure 1. The runway apparatus, with dimensions. 
A

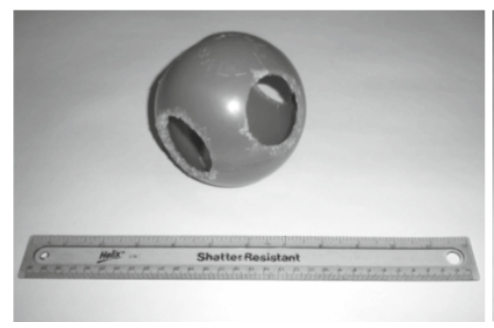

B

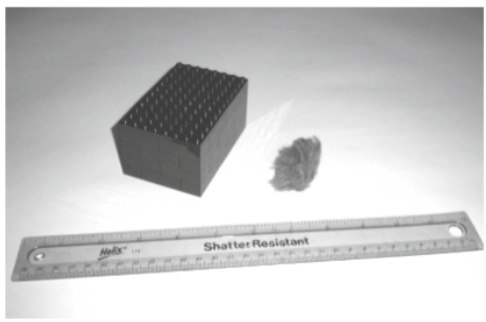

C

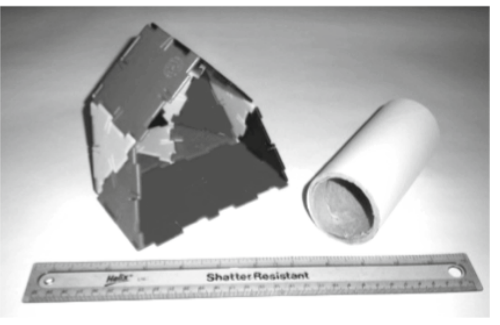

Figure 2. Objects used in Experiment 1. (A) The plastic ball used to train the rats on using the runway and to collect baseline data. (B) The Lego objects (left, large plastic; right, small synthetic fur). (C) The environmental enrichment objects (left, plastic house-shaped shelter; right, cardboard tube).

previous experiment, and an individual Lego brick $(4 \times 2 \times 2 \mathrm{~cm})$ covered in soft synthetic fur, which had been the bottom-ranked object (Williams et al., 2008).

The EE set consisted of objects typically chosen as enrichment objects for rat home cages (Figure 2C). The EE top-ranked object was a plastic house made from flat plastic connectable shapes $(14 \mathrm{~cm}$ wide, $19 \mathrm{~cm}$ long at the longest point, and $11 \mathrm{~cm}$ high at the tallest point), and the EE bottom-ranked object was a small cardboard tube (measuring $12.5 \mathrm{~cm}$ long, $5.5 \mathrm{~cm}$ in diameter; Hanmer, 2008).

All of the apparatus was cleaned using $50 \%$ ethanol solution between each rat's trials, except for the small fur-covered object. Five of these were made, to allow each rat to have one; at the end of the day, these objects were sprayed with a deodorizer (Shaw's Pet Stain and Odour Eliminator) in order to eliminate olfactory cues.

Familiarization and training. Before testing, all of the rats were habituated to and trained in using the runway. A plastic ball was used as the object at the end of the runway - only in the habituation and training phases - as an incentive to motivate the rats to run along the runway.

Initially, in the familiarization phase, pairs of rats were exposed to the runway and plastic ball, for $5 \mathrm{~min}$, to minimize stress and to encourage them to investigate the apparatus. This was repeated on 2 consecutive days. On the following 3 days, the rats were exposed individually to the runway and plastic ball for $5 \mathrm{~min}$.

The training phase followed familiarization to the apparatus: On each of the next 5 days, the rats were individually placed in the start box, the door opened, and timing started. The rats then had $30 \mathrm{sec}$ in which to leave the start box, run the length of the runway, and interact with the object for $5 \mathrm{sec}$. If they were successful, they were placed back in the start box for another trial. They were unsuccessful if they failed to leave the start box, failed to reach the object within $30 \mathrm{sec}$ of the start box door being opened, or failed to interact with the object for at least $2 \mathrm{sec}$ once in the reward box. Three unsuccessful trials in a row resulted in termination of the session for that rat. Eighteen rats were trained on this procedure, and the data were inspected at the end of the 5-day training period. Only rats that had run for at least three successful trials in each of the five training sessions were included in the testing phase (10 rats).

Runway testing. Two replications of testing were carried out with each of the two pairs of test objects. Each replication consisted of counterbalanced days in which the rats saw the familiar plastic ball (baseline), the novel top-ranked object, or the novel bottomranked object in the reward box. The baseline days, with the familiar plastic ball, were included to ensure that the rats maintained their performance levels as testing progressed over the week. The baseline days confirmed that no rat fell below the minimum three runs to the plastic ball on each day. Thus, reduction in motivation in the runway cannot explain differences in performance for the test objects. The criteria used to determine whether a trial was passed or failed on each attempt were the same as those used in training. In each session, a rat was placed in the start box, with an object in the reward box. The door to the start box was lifted, and timing started. The rat then had $30 \mathrm{sec}$ to reach and interact with the object. The rat was allowed to interact with the object for a maximum of $5 \mathrm{sec}$ upon reaching it before being placed back in the start box. This process continued until the rat had failed three trials in succession. The time taken to reach the reward object, the number of trials successfully completed in each testing session, and the time that the rat spent interacting with the object were recorded.

Statistical analysis. After the two replications had been completed with the same objects, a one-way repeated measures ANOVA was conducted on data from each set of objects. This was to determine whether there were significant differences in the data collected in each replication in order to collapse the data across replications.

Two-way repeated measures ANOVAs were conducted for the number of runs, the time it took to reach the objects, and the time spent interacting with the objects. Trial 1 was removed from the analyses of both the number of runs made and the time taken to reach objects, since on Trial 1, the rats could not have known what object they were running toward. Repeated measures Bonferroni $t$ tests were conducted to explore significant interactions.

\section{Results}

The replications with each set of objects did not differ significantly for either the Lego objects $[F(1,9)=3.714$, $p=.086]$ or the EE objects $[F(1,9)=0.938, p=.358]$, and there were no interactions. Therefore, it was assumed that these results show a genuinely replicable effect and that the methodology is reliable. The results were collapsed across replications for the remaining analyses.

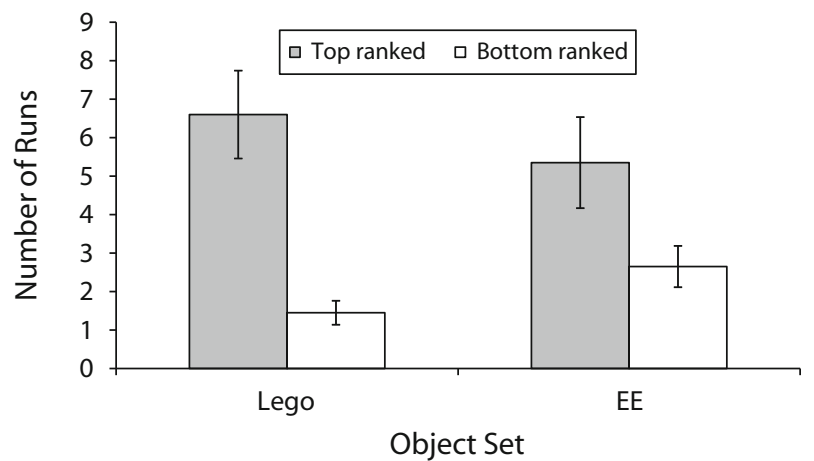

Figure 3. The average number of times the rats ran to reach the objects. Rats ran significantly more times to the top-ranked object than to the bottom-ranked object for both sets. EE, environmental enrichment. 
Table 1

Mean Run Times (With Standard Errors) for Top- and Bottom-Ranked Lego and Environmental Enrichment (EE) Objects Calculated With Failed Runs Excluded or With Failed Run Times Set to 30 sec

\begin{tabular}{|c|c|c|c|c|c|c|c|c|}
\hline \multirow{4}{*}{$\begin{array}{c}\text { Object } \\
\text { Set }\end{array}$} & \multicolumn{8}{|c|}{ Run Times } \\
\hline & \multicolumn{4}{|c|}{ Failed Runs Excluded } & \multicolumn{4}{|c|}{ Failed Runs Set at $30 \mathrm{sec}$} \\
\hline & \multicolumn{2}{|c|}{ Top Ranked } & \multicolumn{2}{|c|}{$\begin{array}{l}\text { Bottom } \\
\text { Ranked }\end{array}$} & \multicolumn{2}{|c|}{ Top Ranked } & \multicolumn{2}{|c|}{$\begin{array}{l}\text { Bottom } \\
\text { Ranked }\end{array}$} \\
\hline & $M$ & $S E M$ & $M$ & SEM & $M$ & SEM & $M$ & SEM \\
\hline Lego & 13.00 & 1.76 & 12.73 & 1.04 & 13.40 & 2.01 & 17.01 & 1.63 \\
\hline $\mathrm{EE}$ & 11.38 & 0.92 & 12.87 & 1.18 & 13.25 & 2.03 & 16.79 & 1.72 \\
\hline
\end{tabular}

Number of runs to objects. Figure 3 shows the number of times the rats ran to reach the objects. There was a significant interaction between object type and ranking $[F(1,9)=5.18, p=.049]$. The rats ran significantly more times to the top-ranked objects $[F(1,9)=24.87, p=$ $.001]$ than to the bottom-ranked objects. Post hoc $t$ tests revealed that there were significant differences between the top- and bottom-ranked objects for both object types [Lego, $t(9)=4.56, p=.001$; EE, $t(9)=3.64, p=.005$ ] In addition to the main effect of ranking, there was also a simple effect for the bottom-ranked objects. There was a significant difference between the Lego and EE bottomranked objects $[t(9)=2.449, p=.037]$, showing that for bottom-ranked objects, the rats ran significantly more times to the EE object than to the Lego object. There was no significant difference in the number of times that the rats ran to the Lego and EE objects overall.

Time taken to reach objects. These data were analyzed twice: First, we excluded trials in which the rats failed to reach or interact with the objects and for which we therefore did not record a time (Table 1), and second, we used a technique employed by other researchers (e.g., Ikemoto \& Panksepp, 1996; Nencini, Graziani, \& Valeri, 1991) in which the maximum time of $30 \mathrm{sec}$ was recorded for failed trials (see Table 1).

Removing failed trials from the data resulted in the exclusion of 1 rat. The left half of Table 1 shows the means for the time taken to reach the objects using this method of analysis. A two-way repeated measures ANOVA was conducted on the data from the remaining 9 rats to determine whether the rats ran faster to the preferred objects than to the nonpreferred objects and also whether the object sets differed. There were no significant differences between the top- and bottom-ranked objects for either object set with the failed trials removed. The right side of Table 1 shows the mean time taken to reach the objects when a maximum time of $30 \mathrm{sec}$ was recorded in place of a failed trial. The ANOVA showed that the rats took significantly longer to reach the bottom-ranked objects than they did to reach the top-ranked objects using this method of analysis $[F(1,9)=7.39, p=.024]$. No other significant effects were found.

Time spent interacting with objects. Figure 4 shows the average time that the rats spent interacting with the objects once they had reached them. A two-way repeated measures ANOVA revealed that there was a significant in- teraction between ranking and object set $[F(1,9)=27.60$, $p<.001]$, a significant difference between the Lego and EE objects $[F(1,9)=26.12, p<.001]$, and a significant difference between the top- and bottom-ranked objects $[F(1,9)=37.88, p<.001]$. Subsequent $t$ tests revealed that the interaction resulted from the rats' spending less time interacting with the bottom-ranked Lego object than with any other object. There was a significant difference between the Lego and EE bottom-ranked objects $[t(9)=$ $5.19, p=.001$ ], with the rats spending longer with the EE bottom-ranked object than with the Lego bottom-ranked object. There was a significant difference between the Lego top- and bottom-ranked objects $[t(9)=6.16, p<$ $.001]$, with the rats spending longer with the top-ranked object, as was predicted.

\section{Discussion}

Overall, this experiment demonstrated that the active runway paradigm is a successful tool for assessing rats' motivational preferences. Clear differences were found between top- and bottom-ranked object types for the number of times that the rats ran, the time that they took to reach the object (when $30 \mathrm{sec}$ was recorded for failed trials), and the time that they spent interacting with the objects (for the Lego set only). However, ceiling effects prevented differences in interaction time for the EE objects from reaching significance. This could be prevented by allowing the rats to interact with the objects for more time (10 sec).

In addition, our results show conflicting evidence regarding the usefulness of the measure of time taken to reach the object, depending on how it is calculated. Using our original data set, with 1 rat excluded for failing to reach the EE objects, we found no significant differences in the run times to any of the objects. This would suggest that this measure is not a reliable way of differentiating between motivational preferences, because the rats run along the runway at the same speed, regardless of the

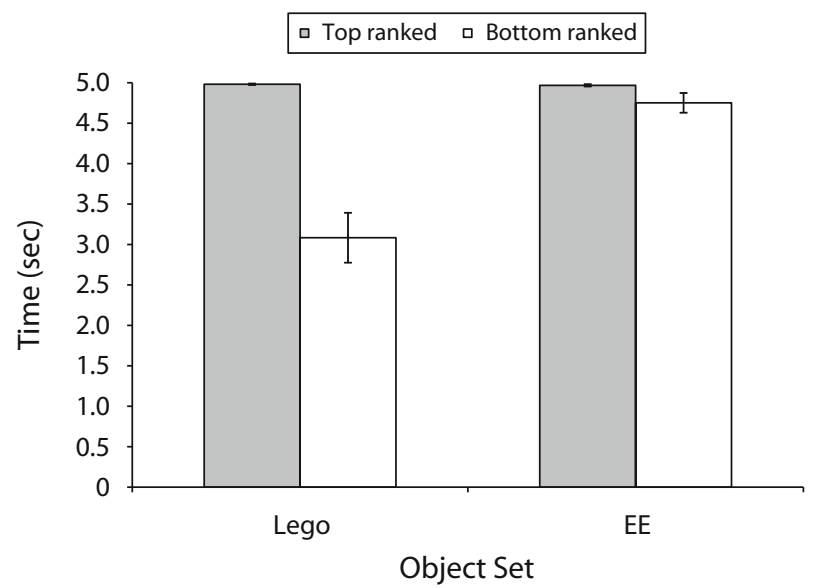

Figure 4. The average time the rats spent interacting with the objects. The rats spent significantly less time interacting with the bottom-ranked Lego object than with any other object. EE, environmental enrichment. 


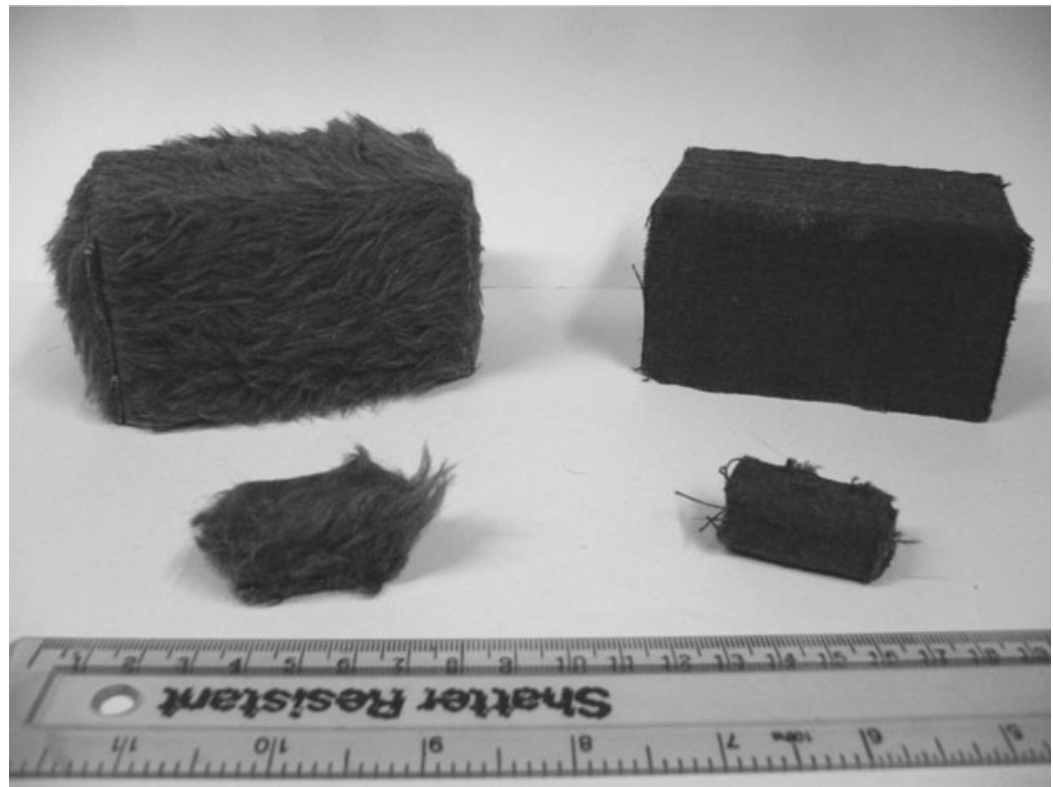

Figure 5. Objects used in Experiment 2. The large and small fur-covered blocks are shown on the left, and the large and small polyester-covered blocks are shown on the right.

object placed in the reward box. Alternatively, when we adapted our design to include a recording of the maximum $30 \mathrm{sec}$ for failed trials, as has been previously done in runway methodologies (e.g., Ikemoto \& Panksepp, 1996; Nencini et al., 1991), we did find a significant difference in the time that the rats took to reach the top- and bottom-ranked objects. However, it is possible that this results from recording $30 \mathrm{sec}$ as the run time for failed trials. Since there were more of these for bottom-ranked objects, this analysis might result in artificially significant data.

There were also differences in time spent interacting when the bottom-ranked object types were compared, but not between top-ranked objects. This suggests that although the top-ranked Lego and EE objects seemed to provide the rats with the opportunity to express highly motivated behaviors to an equal extent (since no differences were found between these objects), the nonpreferred objects permitted different behaviors. The rats ran more times and interacted longer with the bottom-ranked EE object than with the bottom-ranked Lego object. One potential explanation for this is that the bottom-ranked Lego object was a small block covered in synthetic fur (a nonpreferred texture), which allowed only picking up and chewing behaviors, whereas the EE cardboard tube also allowed the rats to put their heads inside the object and to rear up against it. Since the EE and Lego objects also differed considerably in other factors (i.e., texture, size), these might also have contributed to the differences found between the bottom-ranked objects.

A second experiment was conducted to determine whether ceiling effects in interaction time could be eliminated by increasing the interaction time from 5 to $10 \mathrm{sec}$. In addition, a range of objects was used to determine whether the behaviors afforded by the objects or the physical properties of the objects were more influential in determining object ranking in the runway. To achieve this, two large objects and two small objects of identical construction were covered with either polyester or fur. By using Lego objects that had been covered in different fabrics, we were able to consider the size of the object (and therefore the behavior performed) separately from the physical properties of the objects.

The rats used in the second experiment were younger than those used in the first experiment. We therefore might expect the rats in Experiment 2 to demonstrate higher overall levels of motivation for novel objects. However, these predictions are based on within-subjects comparisons for each experiment, so differences in overall levels of motivation were not considered problematic when testing these hypotheses.

\section{EXPERIMENT 2}

\section{Method}

Subjects. Adult male Lister hooded rats $(N=14)$, weighing $350-450 \mathrm{~g}$, were group housed in standard cages under standard conditions ( $n=4$ rats per cage). The cage and housing environments were maintained in the same way as those in Experiment 1.

Apparatus. The apparatus was identical to that in Experiment 1.

Objects. Figure 5 shows the objects tested in this experiment. In order to test objects with intermediate preference, we used four objects that had been ranked with a range of preferences using openfield preference tests from previous work (Hanmer, 2008). We thus used a large polyester-covered block made from Lego bricks $(9.5 \times$ $6 \times 5 \mathrm{~cm}$ ), a large fur-covered block made from Lego bricks of the same dimensions, an individual Lego brick covered in polyester $(4 \times 2 \times 2 \mathrm{~cm})$, and an individual Lego brick of the same dimen- 
A

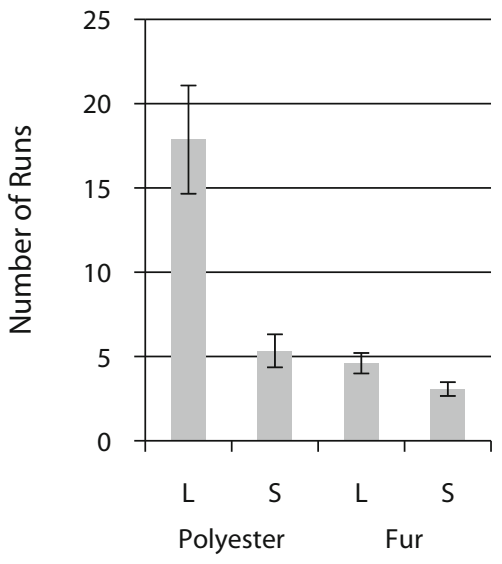

EE Object
B

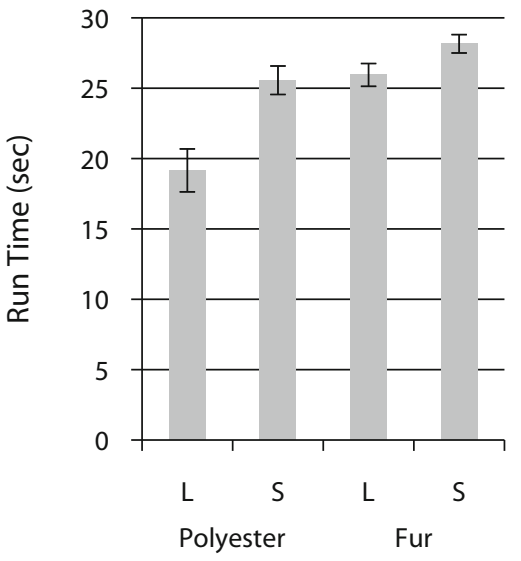

EE Object
C

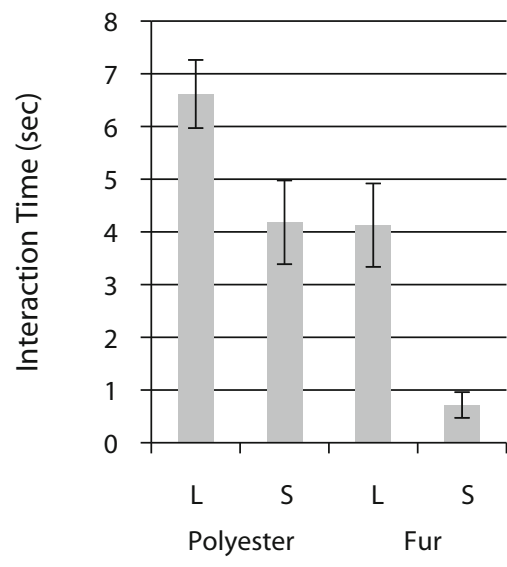

EE Object

Figure 6. Results for Experiment 2. (A) The average number of times the rats ran to reach each of the objects. The rats ran significantly more times to the large polyester-covered object than to any other object. (B) The time taken to reach the objects. The rats ran significantly faster to the large polyester-covered object than to any other object and also ran significantly faster to the small polyestercovered object than to the small fur-covered object. (C) The time spent interacting with the objects. The rats spent significantly less time interacting with the small fur-covered object than with any other object. EE, environmental enrichment; L, large; S, small.

sions covered in fur. Of these, the large polyester-covered object had been top ranked, the small fur-covered object was bottom ranked, and the other two objects had intermediate ranks.

Familiarization, training, and testing. The experiment was conducted in the same manner as was Experiment 1 . All of the animals tested met the criterion for inclusion outlined in Experiment 1.

Statistical analysis. The data were analyzed using a one-way repeated measures ANOVA for number of runs, time taken to reach the objects, and time spent interacting with the objects. As was done in Experiment 1, Trial 1 was removed from the analyses. When analyzing the time taken to run to objects, failed trials were replaced with a maximum time of $30 \mathrm{sec}$. Repeated measures Bonferroni-corrected $t$ tests were conducted to explore significant interactions.

\section{Results}

Figures $6 \mathrm{~A}-6 \mathrm{C}$ show the number of runs (Figure 6A), the time taken to reach the objects (Figure $6 \mathrm{~B}$ ), and the time spent interacting (Figure 6C) for the four objects used in Experiment 2. A one-way repeated measures ANOVA with object as a factor and the number of runs, the time taken to reach objects, and interaction time as measures was conducted. There was a main effect of object for each of the measures [number of runs, $F(1.16,15.08)=17.59$, $p=.001$; run time, $F(1.6,20.81)=17.87, p<.0001$; interaction time, $F(3,39)=14.10, p<.0001]$.

Post hoc tests for the number of runs demonstrated that the rats ran more often to the large polyester object than to any other object $(p<.012)$. No other comparisons reached significance. When the objects were entered in the same rank order as was found in our previous work (Hanmer, 2008), there was a significant linear trend $[F(1,13)=23.22, p<.0001]$, with the rats running more often to the top-ranked objects than to the bottom-ranked objects. For the time taken to run to the objects, there were significant differences between the large polyester object and all other objects $(p<.03)$ and also between the small polyester object and the small fur object $(p=.021)$, and finally, there was a marginal trend for significance between the large and small fur objects $(p=.051)$. As with the number of runs, there was a significant linear trend in the time taken to run to the objects $[F(1,13)=43.57$, $p<.0001]$. The rats took longer to run for the objects of lower ranking.

For interaction time, a post hoc analysis demonstrated significant differences between the small fur object and all other objects $(p<.013)$. No other comparisons reached significance. There was, however, a significant linear trend $[F(1,13)=49.57, p<.0001]$, with the rats interacting longer with top-ranked objects.

\section{Discussion}

In confirmation of our results in Experiment 1, Experiment 2 demonstrated that when the objects were ranked according to the time spent interacting with them in the open field (Hanmer, 2008), there were significant linear trends, with the rats running more often and more quickly to the higher ranked objects.

In Experiment 2, we calculated the time taken to run to the objects by replacing failed run time with a maximum 30 sec (Ikemoto \& Panksepp, 1996; Nencini et al., 1991). As in Experiment 1, this resulted in significant differences between our top- and bottom-ranked objects but also between the intermediately ranked objects. There was a significant linear trend in the time taken to reach the objects when these were ranked according to the time spent in a previous study (Hanmer, 2008). This suggests that the time taken to reach the objects can reflect subtle differences in motivation when calculated in this manner.

In Experiment 2, we reported differences between topand bottom-ranked objects when the rats were allowed a longer interaction time (10 sec in Experiment 2 com- 
pared with $5 \mathrm{sec}$ in Experiment 1). We also demonstrated a highly significant linear trend, with the rats interacting for progressively shorter times according to a descending ranking based on previous interaction times in the open field (Hanmer, 2008). Importantly, no ceiling effects were found when using this length of interaction.

Across all measures, the highest-ranked object was the large polyester block, and the lowest-ranked object was the small fur block. The large fur block and the small polyester block were intermediate in ranking. Thus, neither the size of the object nor the fabric in which it was covered independently predicted ranking, suggesting that the rats were influenced in their preference decisions by both the behaviors that they could perform with an object and the physical properties of that object.

\section{GENERAL DISCUSSION}

The results reveal that the runway paradigm is a suitable methodology to measure the strength of rats' motivational preferences for enrichment objects. The rats were more highly motivated to reach objects with which they had previously chosen to spend more time than they were to reach objects with which they had previously spent little time (Hanmer, 2008; Williams et al., 2008), as was shown by the number of times that they ran to gain access to them, the time taken to run, and the interaction times for the top-ranked versus bottom-ranked objects in Experiment 1 and the linear trends in these measures in Experiment 2. This effect was found to be consistent across replications and with different types of objects, so the runway paradigm is a reliable method for comparing motivational preferences.

All of the objects used in this study had previously been ranked by assessing the amount of time that the rats spent interacting with them (Hanmer, 2008; Williams et al., 2008). The present study has reinforced this object ranking by demonstrating that the same ranking occurs when using a different measure of relative motivational preference. Specifically, across Experiments 1 and 2, we have demonstrated that the number of runs, the method used previously to calculate the time taken to reach the objects, when failed trials are replaced by a maximum time (Ikemoto \& Panksepp, 1996; Nencini et al., 1991), is capable of differentiating the relative ranking of objects, and a 10sec interaction time when rats reach the object at the end of the runway is a suitable parameter to discriminate differences in object ranking.

This active runway methodology could also be used to investigate reward processes associated with motivational preference, because it provides a means to investigate both appetitive and consummatory aspects of the reward process. Using this paradigm, the number of runs and the time taken to reach the object are both measures that can relate to how much the rat wants the object (appetitive component), whereas the time that the rat spends interacting with the object may relate to how much it likes the object (consummatory component). The measurement of both phases of the reward process cannot be made with other measures of preference, such as interaction times, since these are measures only of the consummatory phase. Thus, this methodology provides a more comprehensive assessment of motivation. Pharmaceutical compounds are known to be selectively active in different components of the reward processes (e.g., opioid and cannabinoid agonists and antagonists). By administering these compounds when rats are running for previously ranked objects, the activity of different components of the reward system in these rankings could be investigated. This would increase our knowledge of the role of the reward system in forming motivational preferences for enrichment objects.

\section{Conclusion}

In conclusion, the runway paradigm is a quick and simple method for collecting evidence for motivational preferences for EE objects by determining with which objects rats are most motivated to interact. It might be concluded that objects that produce higher motivational preferences should be added to the cage environment to improve welfare in laboratory rats. This paradigm could also provide a means to investigate reward processes related to object preference.

\section{AUTHOR NOTE}

We thank Elizabeth Gaffan (University of Reading) for her valuable comments on the manuscript. This research was supported by a grant from Universities Federation for Animal Welfare (UFAW) to C.M.W. and P.M.R., and a studentship from the Biotechnology and Biological Sciences Research Council (BBSRC) to L.A.H. (née Scott). Correspondence concerning this article should be sent to C. M. Williams, School of Psychology and Clinical Language Sciences, University of Reading, Earley Gate, Whitenights, Reading RG6 6AL, England (e-mail: claire .williams@rdg.ac.uk).

\section{REFERENCES}

Bradshaw, A. L., \& Poling, A. (1991). Choice by rats for enriched versus standard home cages: Plastic pipes, wood platforms, wood chips, and paper towels as enrichment items. Journal of the Experimental Analysis of Behavior, 55, 245-250.

Burns, R. A., \& Griner, S. E. (1993). Single-alternation patterning in sated, sucrose-rewarded rats. Bulletin of the Psychonomic Society, 31, 35-36.

Burns, R. A., Ziropadja, L., \& DuUric, V. (1984). Effects of liquid sucrose reward reduction on the performance of deprived and nondeprived rats. Journal of General Psychology, 110, 223-228.

Chmiel, D. J., \& Noonan, M. (1996). Preference of laboratory rats for potentially enriching stimulus objects. Laboratory Animal, 30, $97-$ 101.

Flaherty, C. F., Riley, E. P., \& Spear, N. E. (1973). Effects of sucrose concentration and goal units on runway behavior in the rat. Learning \& Motivation, 4, 163-175.

HANMER, L. A. (2008). Rats' interactions with enrichment objects are naturally rewarding: A study of object preference and reward processes. Unpublished doctoral dissertation, University of Reading, England.

Heizmann, V., Jonas, I., Hirschenauer, K., \& Havelec, L. (1998). Choice tests with groups of mice: Nestbox, nesting material and tubes as enrichment items for laboratory mice. Journal of Experimental Animal Science, 39, 43-60.

Ikemoto, S., \& PANksepp, J. (1996). Dissociations between appetitive and consummatory responses by pharmacological manipulations of reward-relevant brain regions. Behavioral Neuroscience, 110, 331345 .

Nencini, P., Graziani, M., \& Valeri, P. (1991). Dapiprazol prevents $\mathrm{U} 50,488 \mathrm{H}$-mediated suppression of preparatory components of drink- 
ing behavior in rats. Pharmacology, Biochemistry, \& Behavior, 40, 125-128.

Patterson-Kane, E. G. (2003). Shelter enrichment for rats. Contemporary Topics in Laboratory Animal Science, 42, 46-48.

Sherwin, C. M., \& Nicol, C. J. (1997). Behavioural demand functions of caged laboratory mice for additional space. Animal Behavior, 53, $67-74$.

Sørensen, D. B., Krohn, T. C., Hansen, H., Ottesen, J., \& Hansen, A. K. (2005). An ethological approach to housing requirements of golden hamsters, Mongolian gerbils and fat sand rats in the laboratory: A review. Applied Animal Behaviour Science, 94, 181-195.

TownsEnd, P. (1997). Use of in-cage shelters by laboratory rats. Animal Welfare, 6, 95-103.

Van de Weerd, H. A., Van Loo, P. L. P., Van Zutphen, L. F. M.,
Koolhaas, J. M., \& Baumans, V. (1998a). Preferences for nest boxes as environmental enrichment for laboratory mice. Animal Welfare, 7, $11-25$

Van de Weerd, H. A., Van Loo, P. L. P., Van Zutphen, L. F. M., Koolhaas, J. M., \& Baumans, V. (1998b). Strength of preference for nesting material as environmental enrichment for laboratory mice. Applied Animal Behaviour Science, 55, 369-382.

Williams, C. M., Riddell, P. M., \& Scott, L. (2008). Comparison of preferences for object properties in the rat using paired- and freechoice paradigms. Applied Animal Behaviour Science, 112, 146-157.

(Manuscript received June 2, 2009;

revision accepted for publication November 17, 2009.) 